\title{
When to abandon the search for an elusive gastrointestinal bleeding source
}

\section{(ㄷ)(구)}

\author{
Author \\ Amnon Sonnenberg ${ }^{1,2}$ \\ Institutions \\ 1 Gastroenterology Section, Portland VA Medical Center, \\ Portland, Oregon, United States \\ 2 Division of Gastroenterology and Hepatology, Oregon \\ Health \& Science University, Portland, Oregon, United \\ States
}

submitted 29.1.2018

accepted after revision 13.3 .2018

\author{
Bibliography \\ DOI https://doi.org/10.1055/a-0605-3418 | \\ Endoscopy International Open 2018; 06: E898-E901 \\ (c) Georg Thieme Verlag KG Stuttgart · New York \\ ISSN 2364-3722
}

Corresponding author

Amnon Sonnenberg, MD, MSc, Portland VA Medical Center P3-GI, 3710 SW US Veterans Hospital Road, Portland, OR

97239, USA

Fax: +1-503-220-3426

sonnenbe@ohsu.edu

\section{ABSTRACT}

Background and study aims In some patients with gastrointestinal bleeding, even multiple consecutive endoscopic procedures fail to achieve lasting hemostasis. The current decision analysis was designed to answer the question of when to continue or abandon a sequence of endoscopic attempts of endoscopic hemostasis.

Materials and methods A decision tree with a threshold analysis was used to model the decision between continued endoscopy or expectant management. A low threshold probability was indicative of a preferred management option.

Results For continued endoscopy to be the favored decision, its probability of success in achieving hemostasis needed to exceed the success probability of expectant management by a greater amount than its costs exceeded those of expectant management. Endoscopic attempts at hemostasis should be discontinued if the costs of endoscopy are high compared with those of expectant management. The endoscopic attempt should be continued, if its probability for achieving lasting hemostasis is high.

Conclusions Such principles are applicable as rule of thumb in managing patients with ongoing chronic gastrointestinal bleeding.

\section{Introduction}

A substantial portion of gastrointestinal endoscopy is devoted to diagnosis and treatment of various potential bleeding sites in the intestinal tract [1,2]. Occasionally, even an extensive search using different types of endoscopic and radiographic procedures fails to identify an unequivocal bleeding source [3]. There is also a subgroup of patients in whom multiple attempts at hemostasis fail to achieve a lasting resolution. For instance, patients with arteriovenous malformations (AVMs) spread throughout the gastrointestinal tract, gastric arteriovenous ectasia (GAVE or watermelon stomach), and radiation proctitis frequently continue to bleed even after multiple sessions of thermo-ablation. Other patients who bleed secondary to chronic use of medical anticoagulation, cannot be taken off their medication because of their continued risk for thromboembolism. An excessive number of futile endoscopic proce- dures may be spent on trying to resolve the gastrointestinal bleeding and achieve lasting hemostasis [4]. If bleeding only occurs at a small rate and patients present with chronic anemia rather than life-threatening episodes of acute and massive blood loss, expectant follow-up represents a management option that is an alternative to continued endoscopic intervention. Such patients can have their blood count monitored on a regular basis and receive occasional blood transfusions as needed. The current decision analysis was designed to answer the question of when to continue or abandon the quest to find and treat an elusive gastrointestinal bleeding source.

\section{Materials and methods}

A decision tree was used to model the choice between a continued endoscopic search versus expectant management with repeat transfusions in patients with gastrointestinal bleeding 


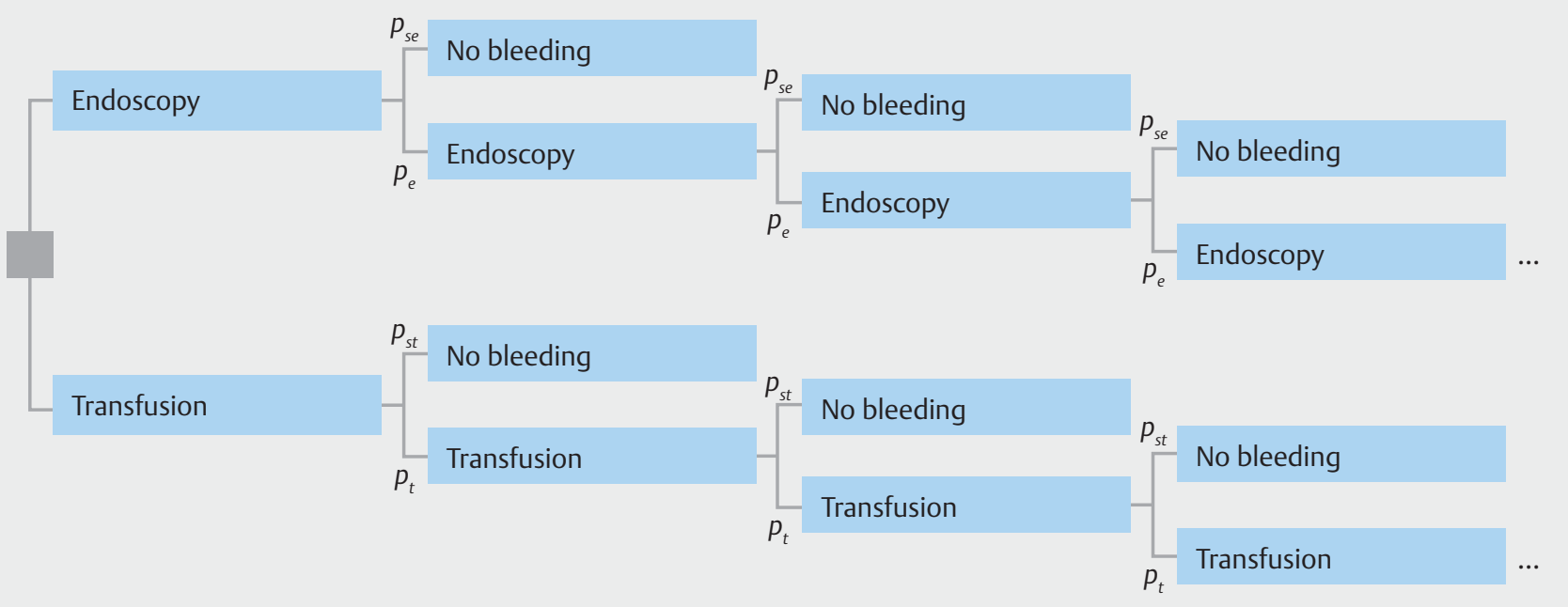

- Fig. 1 Decision tree comparing the outcome of continued endoscopic search versus expectant management with repeat transfusions in a patient with gastrointestinal bleeding from an unknown source.

from an unknown or untreatable source $[5,6]$. In the decision tree, the initial decision node between continued endoscopy and expectant management was represented by a small square on the left ( $\triangleright$ Fig. 1 ). All subsequent outcomes were governed by chance. Each branch following a chance node was assigned a probability value, with all probability values of branches originating from the same chance node adding up to $100 \%$. The outcomes of decisions or chance events were represented by blue rectangles. Each outcome was associated with costs. The outcome of a chance node was calculated by multiplying the individual costs with their respective probability of occurrence and then adding them up at the corresponding branching point. Ultimately, those decisions were favored that resulted in the least costs. Instead of calculating the overall costs, in a threshold analysis one can also calculate the probability values for the possible outcomes that would shift the decision-making against or in favor of one of the two initial decisional options. In general, a low threshold probability was associated with a preferred management option $[7,8]$.

\section{Results}

The decision tree in $\mathbf{\vee F i g . 1} \mathbf{1}$ illustrates the decision in favor of continued endoscopy or continued transfusions. Each endoscopy can be successful and stop the bleeding or be unsuccessful and lead to yet another subsequent endoscopy. The respective probabilities for each set of successful and unsuccessful endoscopy are $p_{s e}$ and $p_{e}$, respectively, where $p_{s e}+p_{e}=1$. A successful outcome with no bleeding is assumed to cost nothing. The cost of the first endoscopy is $E$. The expected cost of the second endoscopy is $p_{e} \cdot E$, the expected cost of the third endoscopy is $p_{e}{ }^{2} \cdot E$, the expected cost of the fourth endoscopy is $p_{e}{ }^{3} \cdot E$, and so forth. The overall expected costs of the decision in favor of endoscopy are:

$$
E+p_{e} \cdot E+p_{e}^{2} \cdot E+p_{e}^{3} \cdot E+p_{e}^{4} \cdot E+\cdots=E \cdot \sum_{n=0}^{\infty} p_{e}^{n}=\frac{E}{1-p_{e}}=\frac{E}{p_{s e}}
$$

A simple rule of analysis was used for the transition from the geometric progression on the left and its summary by the $\Sigma$ sign to the subsequent fraction on the right [9].

The respective probabilities of a successful and unsuccessful transfusion are $p_{s t}$ and $p_{t}$, respectively, where again $p_{s t}+p_{t}=1$. With $T$ representing the cost associated with each individual transfusion, the overall expected costs of the decision in favor of expectant management with continued transfusions are:

$T+p_{t} \cdot T+p_{t}^{2} \cdot T+p_{t}^{3} \cdot T+p_{t}^{4} \cdot T+\cdots=T \cdot \sum_{n=0}^{\infty} p_{t}^{n}=\frac{T}{1-p_{t}}=\frac{T}{p_{s t}}$

For a decision in favor of continued endoscopy as opposed to continued transfusions, the expected costs of the former should be less than those of the latter:

$\frac{E}{p_{s e}} \leq \frac{T}{p_{s t}} \rightarrow \frac{E}{T} \leq \frac{p_{s e}}{p_{s t}}$

The last expression yields a threshold for the success rate of endoscopy to render continued endoscopy the preferred management strategy. For instance, if each endoscopy costs on average five to 10 times more than each transfusion, its associated probability of success (without further bleeding or need for any future endoscopy) also needs to be at least five to 10 times higher than the probability of success associated with expectant management using repeat transfusions.

Assuming, for instance, that endoscopic therapy costs $\$ 1200$ per session and transfusion therapy $\$ 200$, the ratio between the two cost items equals $E / T=6$. Moreover, assuming a probability for successful transfusion therapy of $p_{s t}=5 \%$, endoscopy would need to be associated with an expected success probability of $p_{s e} \geq 6 \cdot 5 \%=30 \%$ to become the preferred management strategy. $\triangleright$ Fig. 2 shows how the threshold for the expected success rate of continued endoscopy varies with the as- 


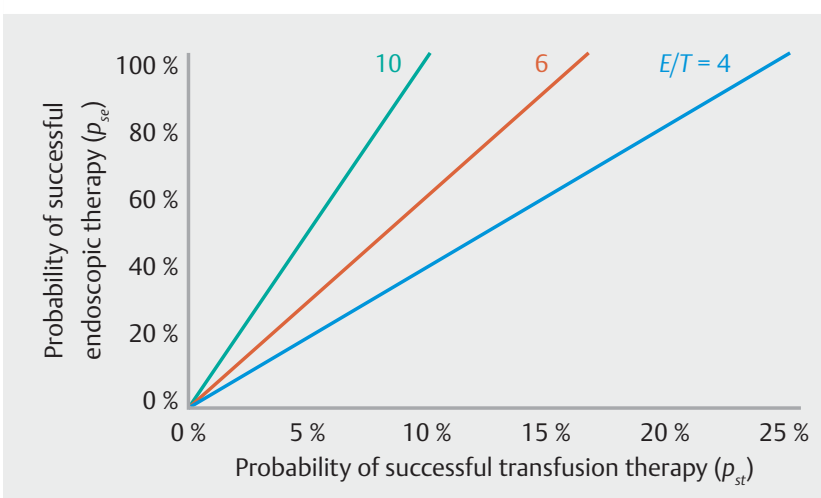

- Fig. 2 Threshold probability for success rate of endoscopy $\left(p_{\text {se }}\right)$ as function of varying success probability for transfusion therapy $\left(p_{s t}\right)$, as well as varying cost ratio for endoscopy over transfusion $(E / T)$.

sumption of success probability for transfusion therapy, as well as different cost ratios for endoscopy over transfusion. The probability for successful transfusion therapy increases the expectation for successful endoscopy. Similarly, increasing costs of endoscopy compared to transfusion also leads to increasing expectation for successful endoscopy.

As a rule, the sequence of continued endoscopies should be abandoned when the expected probability for lasting success associated with the next endoscopy no longer exceeds its threshold probability. In clinical practice, however, one would not necessarily need to engage in such calculation. As indicated by the equation from above, the clinician could simply compare the estimated cost ratio (of continued endoscopy to transfusion therapy) with the expected probability ratio (of the two management options) and abandon endoscopy if the cost ratio exceeds the probability ratio.

\section{Discussion}

A decision tree with a threshold analysis has been used to model when to discontinue a sequence of repeated attempts to diagnose and treat an elusive gastrointestinal bleeding source. The decision in favor or against a continued endoscopic sequence depends on the costs of the two competing management options and their associated chances of success. The analysis reveals that for a positive decision in favor of continued endoscopy, its probability of success in achieving lasting hemostasis needs to exceed the success probability of expectant management by a greater amount than the costs of endoscopy exceed those of expectant management. This outcome of the decision analysis largely confirms what one would expect based on clinical intuition. The underlying principle is readily applicable as a rule of thumb for many situations in management of patients with ongoing chronic gastrointestinal bleeding.

How would the proposed rule of thumb be applied in clinical routine? A low chance of endoscopic hemostasis would argue in favor of expectant management. A high cost of endoscopy and low cost of expectant management would raise the threshold for endoscopic intervention and also speak in favor of expectant management. Old age and serious comorbid conditions would render endoscopy riskier and thus costlier. A low rate of gastrointestinal bleeding and infrequent utilization of transfusion in instances of a shortened life expectancy would reduce overall costs of expectant management and again raise the threshold for endoscopic intervention.

Like any mathematical model of a complex clinical problem, the current analysis had to rely on several simplifying assumptions. For instance, it is assumed that the probability for success or failure stays the same over time, when in reality these probability values may vary among consecutive bleeding episodes. The probability for success is likely to be highest at the beginning and drop after multiple prior attempts that failed at localizing the bleeding source or achieving hemostasis. The analysis does not consider the influence of time and the length of time intervals between consecutive medical interventions. All endoscopic procedures are assumed to cost the same, but different types of endoscopic interventions, such as esophagogastroduodenoscopy, colonoscopy, double balloon enteroscopy, or video capsule endoscopy, with different costs are frequently applied in sequence. Instances of repeat gastrointestinal bleeding could be associated with additional costs resulting from physician visits, laboratory testing or other diagnostics, hospital admission, and potential adverse events of medical intervention. A similar argument would also apply to expectant management with its use of repeat blood transfusions in instances of low blood count. The costs of endoscopy and expectant management used in the current analysis should, therefore, be considered more reflective of average costs associated with individual bleeding episodes leading to repeat endoscopy or transfusion rather than just the costs of the procedure itself or the blood transfusion alone.

\section{Conclusion}

In conclusion, the present decision analysis provides a framework for deciding on when to continue or abandon the endoscopic search for an elusive gastrointestinal bleeding site. On the one hand, the search should be discontinued if the expected costs of additional endoscopic procedures are high compared with expectant management. On the other hand, the search should be continued if the probability for achieving lasting hemostasis is high. This framework may provide useful guidance in managing this common clinical conundrum.

\section{Competing interests}

None

\section{References}

[1] ASGE Standards of Practice Committee, Pasha SF, Shergill A et al. The role of endoscopy in the patient with lower GI bleeding. Gastrointest Endosc 2014; 79: 875 - 885 
[2] Davila RE, Rajan E, Adler DG et al. ASGE Guideline: the role of endoscopy in the patient with lower-GI bleeding. Gastrointest Endosc 2005; 62: $656-660$

[3] ASGE Standards of Practice Committee, Fisher L, Lee Krinsky M et al. The role of endoscopy in the management of obscure GI bleeding. Gastrointest Endosc 2010; 72: 471-479

[4] Woodward Z, Williams JL, Sonnenberg A. Length of endoscopic workup in gastrointestinal bleeding. Eur ] Gastroenterol Hepatol 2016; 28 : $1166-1171$

[5] Sox HC, Higgins MC, Owens DK. Medical Decision Making. 2nd Edition. West Sussex, UK: John Wiley \& Sons; 2013
[6] Petitti DB. Meta-Analysis, Decision Analysis, and Cost-Effectiveness Analysis: Methods for Quantitative Synthesis in Medicine. New York: Oxford University Press; 2000

[7] Pauker SG, Kassirer JP. The threshold approach to clinical decision making. N Engl J Med 1980; 302: 1109-1117

[8] Sonnenberg A. Decision analysis in clinical gastroenterology. Am J Gastroenterol 2004; 99: 163-169, 400

[9] Beyer WM, ed. CRC Standard Mathematical Tables. $26^{\text {th }}$ ed Boca Raton, FL: CRC Press; 1981: 8 